\title{
EDITORIAL: INFECTIOUS DISEASE MODELLING
}

The aim of this Special Issue is to collect together a group of outstanding applied mathematics research articles that provide new insight into our understanding of infectious diseases and infectious disease modelling. The scope of the articles is broad, encompassing both specific applications of modelling to particular examples of infectious diseases, as well as articles that are devoted to the development of more general theoretical insight.

New theoretical work presented by Bartlett and Plank (pp. 3-22) analyses the reproduction number, average final epidemic size and epidemic spreading rate for an SIR model on a scale-free network compared to random networks or a wellmixed population. The effect of spatial heterogeneity in deterministic SIR models is considered by Waters et al. (pp. 23-36) by modelling an epidemic within a population that is treated as discrete patches with the possibility of inter-patch migration. This modelling work analyses the influence of inter-patch movement, exploring how the time for the epidemic to peak and the duration of the infectious period depend on the rate of inter-patch migration. Binder et al. (pp. 37-49) also explore the role of spatial heterogeneity in a hybrid model of infectious disease spread by describing both the stochastic individual-level interactions within the model and the emergent population-level behaviour. Dafilis et al. (pp. 50-63) incorporate a waning state into a deterministic SIRS model to include the effect of immune boosting. By introducing the waning state, the model predicts undamped oscillatory behaviour that is of interest since it is consistent with real observations of multi-annual fluctuations, such as in the case of pertussis (whooping cough). Motivated by the decline in coral reef health and potential threats posed by climate change, Yakob and Mumby (pp. 64-73) extend and adapt the deterministic SIR model framework to include coral diseases and resilience through the inclusion of density-dependent demographic rates. Their modelling indicates the potential for the development of an early warning system for coral reef disease outbreaks as well as pointing to the potential for improving our understanding of how changes in coral population size and composition affect the robustness of the population. Simpson and Roberts (pp. 74-88) present a model of New Zealand's nation-wide meningococcal B disease vaccination programme and compare the outcome to what could have happened without the vaccination programme. A model of Tasmanian Devil Facial Tumour Disease is presented and analysed by Beeton and Forbes (pp. 89-107), with particular emphasis on possible bifurcations in the solution. Finally, the role of cross-immunity in a model of influenza A is examined by Roberts (pp. 108-115), who introduces a two-strain epidemic model where the cross-immunity between the strains is treated as an unknown variable.

Increasing the proportion of papers published in The ANZIAM Journal from the Australian and New Zealand applied mathematics community is a stated goal of the co-chief editors (Editorial, issue 51(3)). We are very pleased that this special issue 
of The ANZIAM Journal has been strongly supported by our community, and will therefore help to meet this goal.

Matthew J. Simpson

Mathematical Sciences,

Queensland University of Technology,

Brisbane 4001, Australia

and

Geoffry N. Mercer

National Centre for Epidemiology and Population Health,

The Australian National University, Canberra,

ACT 0200, Australia

11 December 2012 